\title{
Classificadores nominais em três línguas indígenas da Amazônia brasileira: ampliando tipologias
}

\author{
Nominal classifiers in three Amazonian Indigenous languages \\ of Brazil: enhancing typologies
}

\author{
Ana Suelly Arruda Câmara Cabral ${ }^{1}$ \\ Kaman Kalapalo ${ }^{2}$ \\ Makaulaka Mehinaku Awetí ${ }^{3}$ \\ Sanderson Castro Soares de Oliveira ${ }^{4}$ \\ Uraan Suruí ${ }^{5}$
}

\begin{abstract}
Resumo
Descrevemos três sistemas de classificação nominal presentes em três línguas indígenas brasileiras - Mehináku (família Aruák), Nahukwá-Kalapálo (família Karíb), e Suruí (falada pelos Paitér éy) (família Mondé, tronco Tupí). Com exceção do sistema de classificadores da língua Mehináku, do qual Mori $(2005,2007,2012)$ descreveu alguns aspectos, os outros dois sistemas são aqui descritos pela primeira vez e juntos evidenciam singularidades de sistemas de classificação nominal ainda não considerados em estudos descritivos, históricos ou tipológicos sobre línguas Tupí e Karíb.

Palavras-chave: Classificadores nominais. Bases culturais. Significados metafóricos. Mehináku, Suruí e Nahukwá-Kalapálo.
\end{abstract}

\begin{abstract}
We describe three nominal classifier systems present in three Brazilian Indigenous languages - Mehináku (Aruakan family), Nahukwá-Kalapálo (Karibean family), and e Suruí (spoken by the Paitér éy) (Mondé family, Tupian stock). Except for Mehináku, some aspects of which have been described by Mori $(2005,2007,2012)$, the other two systems are described here for the first time, and together highlight particular characteristics of classifiers systems that have not yet been considered in previous descriptive, comparative or typological studies on the Tupian and Karibean families.

Keywords: Nominal classifiers. Cultural basis. Metaphoric meanings. Mehináku, Suruí and Nahukwá-Kalapálo

${ }^{1}$ Professor associado da Universidade de Brasília, Coordenadora do LALLI e Vicecoordenadora do Núcleo de Estudos Amazônicos da Universidade de Brasília. Pesquisadora em produtividade Científica, CNPq.

${ }^{2}$ Mestre em Linguística pela Universidade de Brasília, professor de língua NahukwáKalapálo no Alto Xingu e pesquisador no LALLI da Universidade de Brasília.

${ }^{3}$ Mestre em Linguística pela Universidade de Brasília, professor de língua Mehináku no Alto Xingu e pesquisador no LALLI da Universidade de Brasília.

${ }^{4}$ Professor adjunto da Universidade do Estado do Amazonas, Centro de Estudos Superiores de Tabatinga e pesquisador no LALLI da Universidade de Brasília.

${ }^{5}$ Graduando em Estudos Interculturais na Universidade de Rondônia, Campus de Ji-Paraná, professor de língua Suruí na Terra Indígena Sete de Setembro e pesquisador no LALLI da Universidade de Brasília.
\end{abstract}




\section{Introdução}

Este artigo trata de três sistemas de classificação nominal presentes em três línguas indígenas brasileiras, cada uma representativa de um agrupamento genético distinto, a língua Mehináku (família Aruák), a língua NahukwáKalapálo (família Karíb) e a língua Suruí, falada pelos Paitér éy (família Mondé, tronco Tupí). As duas primeiras línguas são faladas no Parque Indígena do Xingu, estado do Mato Grosso, e a terceira, na Terra Indígena Sete de Setembro, estado de Rondônia. Com exceção do sistema de classificadores da língua Mehináku, alguns aspectos do qual foram descritos por Mori (2005, 2007, 2012), os demais sistemas são aqui descritos pela primeira vez, e, juntos, evidenciam singularidades de sistemas de classificação nominal ainda não considerados em estudos descritivos e tipológicos sobre as famílias linguísticas Tupí e Karíb. Os dados que lastreiam o presente estudo mostram que (a) os sistemas de classificação descritos são todos representativos dos modos como os falantes concebem os referentes dos nomes de suas respectivas línguas, (b) são sistemas bem definidos e produtivos, e (c) refletem um novo olhar sobre a constituição morfológica e sintagmática dos nomes nessas duas línguas. ${ }^{6}$

\section{Classificação nominal em Suruí}

A língua Suruí possui um sistema de classificação nominal único, quanto a, pelo menos, duas particularidades. Trata-se do menor sistema de classificadores já reportado para uma língua indígena brasileira, assim como para línguas amazônicas. Esse sistema contém apenas duas classes, a classe de nomes, cujos referentes são percebidos ou considerados como redondos, circulares, curvilíneos, esféricos ou circunscritos e a classe dos nomes, cujos referentes não são concebidos como detentores dessas propriedades. O sistema existente em Suruí, embora seja um sistema classificatório baseado em forma, dimensão e extensão com limites ou fronteiras, em que as propriedades salientes são físicas, há nomes de sentimentos e sensações, cujos referentes são associados a essa classe semântica. Chamamos a classe dos nomes com referentes percebidos como redondos, circulares, curvilíneos, esféricos ou circunscritos de Classe dos $\operatorname{Cir}$ (cunscritos), e a outra, de Classe dos $N($ ão)-Cir(cunscritos). A classe Cir é a classe marcada; todos os seus elementos são marcados pelo enclítico $a h$ 'Cl(assificador).Cir(cunscrito)', enquanto que os elementos da classe

\footnotetext{
${ }^{6}$ Abreviaturas usadas: ABS. = absoluto; ARR. = Arredondado; ARQ. = Arqueado; CIR. $=$ Circunscrito; CIRCUN. = circunstância; CL. = Classificador; CURV. = Curvilíneo; FIN. = Fino, sinuoso, em forma de espiral; INV.= Invólucro; REF. = Referência; MRP = Mediador de relação de posse; N-CIR: $=$ Não-circunscrito; NLZD $=$ Nominalizador; PEL. $=$ Pele; SUP.PLANA $=$ Superfície plana; $1=$ Primeira pessoa do singular.

${ }^{7}$ Não foram representados os tons nas palavras do Suruí.
} 
dos $N($ ão)-Cir(cunscritos) não recebem esta ou outra marca. As duas classes possuem nomes de vários campos semânticos - partes do corpo, animais, objetos pessoais, elementos da natureza, dentre outros.

Exemplos de nomes com referentes da classe dos circunscritos são os seguintes:

Nomes de partes do corpo
1) intestino grosso
gor ah pep ah
2) rim
merpi kap ah
3) reto
ĩwã ipo ahp sak ah
4) pulmão
agõa serep ah
5) aorta
agõa kap etapo ah
6) seio (mulher)
walet nom ah
7) bico do peito
nom ah ipo
8) peito (homem)
oy nom ah
9) pênis
labor ah
10) umbigo
gonio ah
11) bunda
wepiyõm ah (wepi trazeira yõm carne)
12) cintura
gatap ah
13) moleira
anar ah dog dog ah
14) olhos
alakap ah
15) pupilas
alakap ah kap
16) íris
alakap ah kap apirup
17) dentes molares
ikahp beti ah
18) dentes cisos
ikahp sag ah
19) piolho grande (espécie)
g̃ip mo ah
20) corcunda
abe ah

Na construção nom ah ipo, (exemplo 7), o que é marcado como circunscrito é nom 'peito', mas não a locução ‘bico do peito'. Por outro lado, no exemplo 13, tanto anar 'cabeça', quanto a locução que significa 'moleira' recebem a marca $a h$. Esses dados evidenciam uma classificação de referentes, em que os nomes são constituídos apenas de um tema ou de uma locução. No caso de locuções, os temas nominais constituintes internos podem ser cada um deles marcados por ah, como nos exemplos 1 e 13. 
O intestino grosso (ex. 1, repetido em seguida como ex. 21) é cilíndrico e circunscrito, enquanto que o intestino delgado é visto como não cilíndrico e não circunscrito.
21) intestino grosso
gor ah pep ah
22) intestino delgado
gor ah xiter

O contraste entre dentes molares e os terceiros molares ou dentes do siso com os dentes caninos e incisivos mostram a divisão dos dentes em duas classes, a dos molares e a do siso pertencem à classe dos $\mathrm{Cir}$, e a dos caninos e incisivos, à classe dos $N$-Cir:
23) dentes molares
ikahp beti ah
24) dentes do siso
ikahp sak ah
25) dentes caninos
ikahp ewato
26) dentes incisivos
ikahp abi

Nomes de animais

$\begin{array}{lll}27) & \text { porakê } & \text { gopam ah } \\ \text { 28) } & \text { tucunaré } & \text { nambe ah } \\ 29) & \text { esp. de jaú } & \text { morip ah } \\ 30) & \text { bagre } & \text { yoir ah } \\ 31) & \text { cascudo } & \text { likĩã ah } \\ \text { 32) } & \text { tipo de cascudo } & \text { ososo ah }\end{array}$

Nomes de plantas, suas partes e seus frutos ou frutas
33) fruta
ihp ah
34) mamão
ibok ah
35) ingá
morili ah
36) banana
bokop ah
37) cacau
akop ah
38) cupuaçu
akop gam ah
39) nós das árvores
ihp kap ah
40) semente de capim
payaih kap ah
41) palmito
pasap anar ah 


$\begin{array}{lll}\text { 42) } & \text { fruta da seringa } & \text { bar kap ah } \\ 43) & \text { ouriço da castanha } & \text { mag̃ap ah } \\ \text { mayor ah } \\ \text { 44) } & \text { tucumã } & \text { borka ah } \\ 45) & \text { certa fruta } & \text { so ah } \\ 46) & \text { cará } & \text { watxĩg ah } \\ 47) & \text { batata doce } & \text { abi ah } \\ 48) & \text { pama } & \text { yobar ah } \\ 49) & \text { pupunha } & \text { ipo ah } \\ 50) & \text { algo cilíndrico } & \text { napo ah }\end{array}$

O morfema ah do Suruí vem do PT **?a 'cabeça', 'coisa redonda', e a palavra para 'fruta' em Suruí é originária da combinação de PT *iip 'pau' com **?a, *?ip?a 'fruta', cujo reflexo atual em Suruí é ihp ah. Entretanto, sincronicamente, a palavra fruta do Suruí se comporta como uma forma lexicalizada, embora com a semântica do reflexo **?a preservada. Os exemplos 40 'semente de capim' e 42 'fruta da seringa' evidenciam a função meramente classificatória do morfema ah, mancando respectivamente os referentes de payaih kap [[[capim] [semente]] CIR ]e bar kap [[[seringa] [semente]] CIR], como pertencente à classe dos circunscritos.

Nomes de aves
52) mutum
wakoy ah
53) pato ipey ah
54) rolinha dori ah
55) awar ah
Papagaio

Outros animais

56) jaboti

amõ ah

57) tanajura mor ah

58) sapo (gen.) koroy ah

59) abelha europa warwar ah

Nomes de cores

60) vermelho iyohp ah

61) preto xiip ah 
$\begin{array}{ll}\text { 62) branco } & \text { ikir ah } \\ \text { 63) amarelo } & \text { parakap ah }\end{array}$

Os nomes de cores constituem a única classe semântica em que todos os elementos são marcados por $a h$.

Nomes de artefatos

64) vasilha de carregar água ih ah

65) panela grande itxir ah

66) fio de rede enrolado $\tilde{1}$ ah

67) rolo de barbante gop tapo ah

68) machado nambe ah

69) machado grande nambe kot ah

70) ninho lap ah

O exemplo 64 mostra que, em um tempo anterior, o morfema ah do Suruí tinha uma função derivacional, como é também o caso do exemplo 33, discutido anteriormente. Entretanto, palavras como ihp ah 'fruta' e ih ah 'tipo de panela' são exemplos de lexicalizações. ih ah lexicalizou-se como um tipo de panela, entre os diversos tipos de panelas da cultura material Suruí.

Nomes de elementos da natureza
71) pedra
ixa ah
72) pedra branca ixa ah kir
73) o interior do céu gani ah

Nomes de formas geométricas, toda forma que tem lados, bordas

74) kabet ah objeto bidimensional ou tridimensional

75) superfície lisa xirip ah

76) algo.redondo patakap ah

Nomes de tempo e lugar

77) lugar onde alguém passa fome so ganem ah

78) Entrada perãy am ah (lugar onde bifurca) 
79) lugar no meio do caminho onde

metaret ah um grupo de Paiter ficava antes da festa, sem se misturar com o outro grupo

80) final, lugar terminal, tempo terminal

81) cemitério, onde os Paiter terminam no final da vida

82) final do céu

83) lugar no meio do caminho onde um grupo de Paiter ficava antes da festa, sem se misturar com o outro grupo.

84) espaço central da aldeia gani om ah

om ah

Paiter om ah (onde somem os Paiter) metaret ah

me ah

Nomes de sensação; de características físicas e morais

85) alegria

86) mentiroso (mentira merãye)

87) debilitado

88) teimoso

89) sujo

90) gordo

91) barrigudo kuy ah

perãy ah

xerep ah

ximaxor ah

pesok ah

ipoy ah

xaga ah

Outros sistemas de classificação nominal classificatórios de referentes de nomes, que marcam sintagmas nominais, foram descritos para o Káro (fam. Ramaráma, tronco Tupí) por Gabas Jr. (1999) e para o Mundurukú (fam. Mundurukú) por Crofts (1973, 1985) e por Gomes (2009), dentre outros. O classificador $a h$ do Suruí Paitér é cognato do lexema classificador 'a 'cabeça' do Mundurukú e do morfema classificador 'a do Káro. O classificador do Suruí ah tem uma origem nitidamente lexical, o que pode ser constatado na constituição da palavra para fruta, ihp ah, originalmente [[árvore] [fruta]], embora $a h$ sozinho não tenha, na atualidade, o significado de 'fruto', diferentemente do seu cognato em Mundurukú (fam. Mundurukú, tronco Tupí), o lexema 'a 'cabeça', por 
exemplo, existe enquanto lexema e funciona como classificador de referentes de outros nomes (cf. Gomes 2009:8). Os lexemas classificadores do Mundurukú se combinam também com verbos transitivos, funcionando como um sistema de concordância, mas isso não ocorre com o morfema classificador $a h$ do Suruí. Nesta língua, o morfema ah marca apenas sintagmas nominais, como mostram os exemplos seguintes:

92) machado

93) machado grande

94) pedra

95) pedra branca

96) nós das árvores

97) semente de capim

98) palmito

\author{
nambe ah \\ nambe kot ah \\ ixa ah \\ ixa ah kir \\ ihp kap ah \\ payaih kap ah \\ pasap anar ah
}

Há evidências de que a língua Suruí tenha desenvolvido no passado um sistema de classificadores nominais com mais elementos, dentre os quais um classificador de referentes vistos como oblongos/cilíndricos po (sobo 'cobra', napo 'cipó', txapo 'fio', ipo 'algo cilíndrico', lapom 'rabo'), outro classificador de referentes, sendo estes vistos como foliformes/filiformes hin/din ( $m o$ hin 'folha', nar hin 'cabelo', so-din 'caderno', lab lin 'telha', 'palha', inur hin 'pena de pássaro'), dentre outros (Cabral et al., em preparação). Por outro lado, os dados revelam que esse sistema não teria evoluído, fixando-se apenas um único classificador $a h$.

Outro fato que ressaltamos aqui é o de que o classificador $a h$ ativo em Suruí Paitér, é também ativo em outras quatro línguas da mesma família -Zoró, Gavião, Cinta-Larga e Aruá. Alguns dados que nos forneceram professores nativos dessas línguas revelam que esse sistema está presente na família Mondé, como um todo.

É mister salientar ainda que um sistema análogo de classificação nominal é encontrado na língua Tuparí, família Tuparí. Dados analisados por Raul Tuparí, Cabral e Isidoro mostram que, no Tuparí, os referentes dos nomes são também vistos ou como circunscritos ou como não circunscritos (Cabral et al. idem).

\section{Nahukwá Kalapálo}

Em Nahukwá Kalapálo, uma das duas línguas da família Karíb falada no Alto Xingu (Kaman Kalapálo 2014), há dois sistemas de classificação nominal. Um deles classifica semanticamente referentes de nomes de acordo com a sua 
forma, textura, dimensão, organização em conjuntos, e quanto a características de funções sociais. Este sistema é constituído de morfemas sufixais que se combinam com temas nominais, exclusivamente quando estes entram em uma relação de posse com um determinante. O outro sistema consiste em um conjunto de lexemas genéricos que mediam relações de posse, envolvendo nomes cujos referentes são absolutos. Há ainda em Nahukwá-Kalapálo posposições que distinguem complementos cujos referentes são líquidos, como é também encontrado em outras línguas Karíb, (cf. Derbyshire 1999:42-43), mas aqui descrevemos apenas os dois primeiros sistemas de classificadores.

\section{Morfemas Classificadores}

São seis os morfemas classificadores identificados até o presente em Nahukwá-Kalapalo. Esses morfemas classificam referentes de nomes quanto à forma, textura, dimensão, organização em conjuntos e quanto a características de funções sociais.

Classificador -tsy. Classifica seres vistos como capilares, desfiáveis, compostos de fios, pelos, ou que nascem como pelos, os quais podem também formar um aglomerado, um feixe, ocupando um espaço definido de uma área:

99)

u-ikypí-tsy
1-barba-CL.PELos
'minha barba'

101) u-ijatapí-tsy

1-sovaco- cl.PELOS

'meu sovaco'

103) u-inapí-tsy

1-nariz-CL.PELOS

'pelo do meu nariz'

105) u-inpuhí-tsy

1-pubis-CL.PELOS

'meu pelo pubiano'
100) u-npyngaí-tsy

1-bracelete-CL. PELOS

'meu bracelete'

102) Uraan byngaí-tsy

Nom.prop. bracelete-CL. pel

'bracelete de Uraan'

104) u-imyhuí-tsy

1-acne-CL. PELOS

'minha acne'

106) u-lakumí-tsy

1-tornozeleira-CL.PELOS

'minha tornozeleira'

O exemplo 104 'minha acne' recebe o classificador -tsy, por nascer como pelos, despontando na pele, segundo os Nahukwá-Kalapálo consultados por Kaman Nahukwá. 
Classificador - gu. Classifica referentes percebidos como arqueados, convexos:
107) u-ehú-gu
1-canoa-CL.arq
'minha canoa'
108) u-táho-gu
1-faca-CL.arq
'minha faca'

\author{
109) u-tahakú-gu \\ 1-arco-CL.arq \\ 'meu arco' \\ 110) u-túhu-gu \\ 1-costas-CL.arq \\ 'minhas costas'
}

O exemplo seguinte mostra como a cultura Nahukwá-Kalapálo percebe o cabelo. É algo que contorna as curvas da cabeça, da testa, dos ombros, dos seios, enfim, contorna certas curvas do corpo. O mesmo se dá com os humores líquidos, como o sangue, a lágrima, o suor. A urina é também classificada na classe dos convexos arqueados porque, mesmo quando expelido sem contato com o corpo, o seu jato tem forma curva, arqueada, convexa.
111) u-ngákahu-gu
1-cabelo-CL.arq
'meu cabelo'
113) u-íngaku-gu
1-lágrima-CL.arq
'minha lágrima'
112) u-gítsu-gu
1-urina-CL.arq
'minha urina'

Os referentes dos nomes para animais de estimação e filho (de homem e de mulher), por exemplo, são culturalmente vistos como arqueados, pois são abraçáveis, colocados em tipoias:
114) u-tólo-gu
1-xerimbabo-CL.ARQ
'meu bicho de estimação'

115) u-mú-gu
1-filho-CL.ARQ
'meu filho (de homem)'

Classificador $-n g y \sim-n g u$. Classifica referentes percebidos como protetor, invólucro, que vestem, que abrangem, que abarcam, que tomam conta de algo ou alguém. Observe-se que os nomes 'doença', 'sono' e 'calor' pertencem a essa classe, pois são vistos como estados e sensações que tomam conta, abarcam ou possuem os seres. 
116)
u-í-ngy
1-roupa-CL.INV
'minha roupa'

118) u-kugíhy-ngy

1-doença-CL.INV

'minha doença'
117) u-átu-ngu

1-calor-CL.INV

'meu calor'

119) Ø-u-ítu-ngu

1-Sono-CL.INV

'meu sono'

O nome "braço" também é visto como invólucro, pois é o que abraça.

120) u-îku-ngu

1-braço-CL.INV

'meu braço'

A alomorfia $-n g y \sim-n g u$ é fonologicamente condicionada. A vogal $y$ do alomorfe -ngy se harmoniza com um $u$ precedente: ${ }^{8}$

O tema para roupa se combina com - $n g u$, muito provavelmente por analogia a sapato, pois os dois são vestimentas, uma do pé e outra do corpo.

Classificador -sy. Classifica referentes vistos como finos, os quais podem ser também sinuosos ou em forma de espiral.
121) u-hiu-sy
1-fio-CL.FIN
'meu fio'
123) u-kohoda-sy
1-corda-CL.FIN
'minha corda'
125) u-tíhagi-sy
1-arraia-CL.FIN
'minha arraia'
122) u-igáti-sy
1-lábio-CL.FIN
'meu lábio'

124) u-húti-sy

1-perna-CL.FIN

'minha perna'

O nome para 'arraia' (ex. 125) recebe o classificador -sy, por ser fino seu ferrão.

\footnotetext{
${ }^{8}$ Processo similar foi descrito para o Kuikúro por Franchetto (1995:71-72) "As formas do sufixo REL -gu e -gü são fonologicamente condicionadas. A primeira é determinada por assimilação, pelo espraiamento do traço [labial] da última vogal do radical, enquano -gy é a forma default)." Embora os morfemas do Kuikúro cognatos do Kalapálo não sejam analisados por essa autora como constituído da semântica que descrevemos para o Kalapálo.
} 
A pintura corporal é feita por meio de traços finos e/ou sinuosos, assim como são sinuosos, finos e longilíneos o bico do beija-flor e o rabo do macaco, por exemplo.

126) u-ikú-sy

1-pintura-CL.FIN

'minha pintura'

127) u-kajy-sy

1-macaco-CL.FIN

'meu macaco'
128) u-pynhy-sy

1-beija-flor-CL.FIN

'meu beija-flor'

Objetos emprestados de outras culturas também são classificados na classe - $s y$ se sua característica saliente for a de possuir fio, como ocorre com as palavras adotadas do português 'celular' e 'computador', que entram na classe -sy por possuírem fios:
129) u-celula-sy
1-celular-CL.FIN
'meu celular'
130) u-computado-sy
1-computador-CL.FIN
'meu computador'

Classificador -gy. Classifica as coisas, sentimentos e sensações arredondadas, circulares e/ou circunscritas.
131) u-ntá-gy
1-boca-CL.CIR
'minha boca'
136) u-ý-gy
1-machado-CL.CIR
'meu machado'
132) u-ánke-gy
1-maracá-CL.CIR
'meu maracá'
137) u-angatý-gy
1-seio-CL.CIR
'meu seio'
133) u-hánga-gy
1-orelha-CL.CIR
'minha orelha'
138) u-íti-gy
1-rede-CL.CIR
'minha rede'
134) u-bóla-gy
1-bola-CL.CIR
'minha bola'
139) u-ãỹ-gy
1-piolho-CL.CIR
'meu piolho'
135) u-inatá-gy
1-nariz-CL.CIR
'meu nariz'


Classificador -ty. Classifica certas partes do corpo e a palavra para nome. Agrupa referentes que simbolizam partes do corpo de importância para a cultura Nahukwá, assim como o nome que recebe cada um. Glosamos esse classificador como Classificador de referência -CL.REF.
140) u-inhuti-ty
1-testiculo-CL.REF
'meu testículo'
145) u-ká-ty
1-gordura-CL.REF
'minha gordura'
141) u-áti-ty
'parte de trás do meu joelho'
1-parte.de.trás.do.joelho-CL.REF
146) u-ímy-ty
1-cara-CL.REF
'minha cara'
142) u-hi-ty
1-Coxa-CL.REF
'minha coxa'
147) u-hóni-ty
1-umbigo-CL.REF
'meu umbigo'
143) u-híni-ty
1-meio da testa-CL.REF
'meio da minha testa'
148) u-éngi-ty
1-veia-CL.REF
'minha veia'
144) u-itsoji-ty
1-canela-CL.REF
'minha canela'
149) u-íti-ty
1-nome-CL.REF
'meu nome'

\section{Sobre a classificação de certos nomes de parentesco}

É interessante notar que os termos que nomeiam relações de parentesco estão distribuídos na maioria das classes, mas o que os associam às suas respectivas classes são motivações de natureza metafórica.

Termos de parentesco como "avô", "irmão" e "cunhado" entram na classe -gy 'circunscrito, redondo'.

150) u-ngaũpy-gy

1-avô-CL.CIR

'meu avô'

151) u-hametí-gy

1-cunhado-CL.CIR

'meu cunhado'
152) u-hisuỹ-gy

1-irmão-CL.CIR

'meus irmãos'

Note-se que "irmãos", incluindo homens e mulheres, pertencem também à classe - $g y$, pois são vistos como conjunto, como circunscrito. 
Já "minha avó" entra na classe dos capilares, peludos, que nascem como pelos.

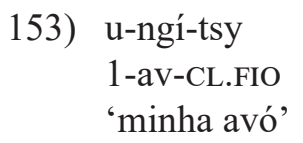

Ressaltamos que a avó é guardada como uma imagem de mulher tradicional, com cabelos grandes e zeladora dessa tradição, assim como do tratamento do cabelo de acordo com os rituais, por exemplo, o ritual do luto, em que se deixa o cabelo crescer e só pode ser cortado, assim como a tradicional franja, depois de passado o luto. É ela também que transmite o conhecimento do tecer fios.

\section{Nomes genéricos classificadores}

O segundo sistema de classificadores do Nahukwá-Kalapálo é constituído de oito nomes relativos que funcionam como classificadores genéricos, conhecidos na literatura como classificadores genitivos (cf. Grinevald, 2000, 2002), os quais, em Nahukwá-Kalapálo mediam relações de determinação nominal envolvendo nomes absolutos.

-ingy 'invólucro'

154) u-íngy camisa

1-INV. CIR camisa

'meu invólucro, camisa'

155) u-húti-sy ingy jeans

1-perna-cl INV jeans

'invólucro da minha perna, jeans'
156) u-túpy-gy ingy tênis 1-pé-CL.CIR INV tênis 'invólucro do meu pé, tênis'

157) u-ínhaty-gy ingy luva 1-mão-CL.CIR INV luva (coberta da minha mão) 'minha luva'

-úgu 'alimento que se come mastigando'

158) Ø-úgu kíne

1-comida beiju

'meu beiju'

(beiju que vou comer)
160) Ø-úgu intse

1-comida pequi

'meu pequi'

(pequi que vou comer)

159) Ø-úgu nhinga

1-comida castanha de pequi

'minha castanha'

(castanha que vou comer) 
-óku 'genérico de mingau'

161) u-óku-gu kuigiku

1-mingau-CL.ARQ mandioca brava

'meu mingau de mandioca brava'

Mingau recebe a marca de arqueado, porque dentro do recipiente ele fica em uma forma arqueada.

162) u-óku-gu tilisinhy

1-mingau-CL.ARQ mandioca

'meu mingau de beiju'

163) u-óku-gu tyhekinga

1-mingau-CL.ARQ mingau de milho 'meu mingau de milho'

165) u-óku-gu suco

1-mingau-CL.ARQ suco

'meu suco'

166) u-óku-gu meisahalu

1-mingau-CL.ARQ mandioca doce 'meu mingau de mandioca doce'

164) u-óku-gu intsene

1-mingau-CL.ARQ polpa de pequi

'meu mingau de pequi'

-ngitsy 'comida que se chupa ou se lambe'

167) u-ngítsy-gy manga

1-chupado-CL.CIR manga

'minha manga'

169) u-ngítsy-gy laranja

1-chupado-CL.CIR laranja

'minha laranja'

168) u-ngítsy-gy katuga

1-chupado-CL.CIR mangaba

'minha mangaba'

170) u-ngítsy-gy kuhugu

1-chupado-CL.CIR macaúba

'minha macaúba'

-ínhango se refere a todas as comidas:

171) u-ínhango kuigiñu

1-comida polvilho

'meu alimento básico polvilho'

-ótu 'comida já servida, já no prato'

172) u-ótu kanga

1-comida peixe

'minha comida peixe' 
-tolo 'animal de estimação, xerimbabo'. -tolo classifica todo animal que pode servir de xerimbabo para os Nahukwá-Kalapálo

173) u-toló-gu katsogo

1-xerimbabo-CL.ARQ cachorro

'meu bicho de estimação cachorro'

174) u-toló-gu tahitse

1-xerimbabo-CL.ARQ arara

'meu bicho de estimação arara'

-engiko 'genérico coisa'. Este genérico classifica coisas em geral. Note-se que o genérico -engiko é sempre marcado por - $g u$, pois o referente passa a pertence, como os animais de estimação e tudo que se tem em sua posse.
175) u-engiko-gu calça
1-coisa-CL.ARQ calça
'minha coisa calça'
176) u-engiko-gu hygé
1-coisa-CL.ARQ flecha
'minha coisa flecha'

177) u-engiko-gu computador 1-coisa-CL.ARQ computador 'minha coisa computador'

178) u-engiko-gu tênis

1-coisa-CL.ARQ tênis

'minha coisa tênis'

\section{Nominalizações de objeto}

Além dos nomes classificadores genéricos, verbos transitivos nominalizados por meio de -tyhy, -tsy e - $p y$ funcionam como nomes genéricos nessas estruturas.

179) ũ-npule-tyhy-gy kanga 1-assar-nom-CL.CIR peixe 'meu assado peixe'

180) ũ-npule-tyhy-gy agaty 1-assar-nom-CL.CIR jacu 'meu assado jacu'

181) ũ-npule-tyhy-gy kajy 1-assar- nom-CL.CIR macaco 'meu assado macaco'
182) u-nkaty-tsy-gy agaty

1-fritar- nom-CL.CIR jacu

'meu jacu frito'

183) u-ngilante-py-gy kanga

1-cozinhar-nom-CL.CIR peixe

'meu cozido peixe'

184) u-ngilante-py-gy angisa

1-cozínhar-nom-CL.CIR batata

'meu cozido batata' 
O nome genérico 'coisa chupada', assim como a maioria dos nomes genéricos obtidos por meio de nominalização combinam-se, por sua vez, com o classificador - $g y$ 'circunscrito', por se tratar de porção delimitada. Já o nome genérico -ngiko 'coisa, pertence' combina-se com o classificador - gu 'arqueado, como é o caso também do nome genérico para 'mingau', o primeiro por ter como referente coisas possuíveis, o segundo por serem os referentes líquidos, como o sangue, a lágrima, entre outros.

Nem todos os nomes em Nahukwá-Kalapálo recebem classificadores. Dentre os quais destacamos as nominalizações por meio do nominalizador -ho 'nominalizador de nomes de circunstância'.

185)

u-aka-ntóho
1-banco-nom

'meu banco'

186) u-tuhinhá-ho

1-roça-NOM

'meu roçado'

187) u-eku-tóho

1-porto-NOM

'meu porto'

188) u-nakangó-ho

1-banhar-NOM

'meu lugar de tomar banho'
189) u-ynky-tóho

1-dormir-NOM-

'meu lugar de dormir'

190) u-tahehi-ó

1-escrever-NOM

'meu lugar de escrever'

191) u-tinguhe-tóho

1- estudar-NOM

'meu lugar de estudo'

O nome para 'sogro/sogra' expressa o respeito que o genro ou a nora sente por eles, e têm como tema uma nominalização por meio de -toho $\sim-h o$ :

192) u-hytiso-ho

1-respeitar-nom-CL.CIRCUN

'meu sogro ou minha sogra', literalmente 'meu lugar de respeito'

O genérico -ótu ‘comida já no prato', na construção em 172, repetida aqui como 193, não recebe classificador, pois 'peixe' é indefinido quanto à forma, dimensão, mas no exemplo 179, repetido aqui como 194, o nome genérico 'assado' vem marcado pelo classificador - gy 'circunscrito', por se tratar de uma porção definida, delimitada. Também não recebem classificadores os nomes genéricos -ínhango 'alimento genérico' e -úgu 'alimento que se come mastigando', respectivamente nos exemplos 171 e 158, 159 e 160, por razões análogas. 
193) u-ótu kanga

1-comida no prato peixe

'minha porção de peixe (já no recipiente)'

194) ũ-npule-tyhy-gy kanga

1-assar-nom-CL.CIR peixe

'meu assado peixe'

\section{Classificadores em Mehináko}

O último sistema de classificadores que apresentamos aqui é o encontrado na língua Mehináku, inicialmente descrito por Mori (2005, 2007, 2012). Mori identificou oito classificadores em Mehináku - -tari 'esférico redondo', -ti 'semente, forma cilíndrica', -pana 'foliforme', - $p i$ 'linear', -já 'líquido', -pe 'massa', -kana 'côncavo', e -ka 'plano'. Somam-se a esses oito classificadores mais dois, identificados por nós em nossa pesquisa, chegamos a um total de dez morfemas sufixais classificadores, que classificam referentes de nomes de animais, de objetos e de plantas, e outros referentes, de acordo com as propriedades culturalmente salientes destes segundo o modo de ver o mundo do povo Mehináku. Separamos aqui esses classificadores em dois conjuntos, considerando suas características mais salientes: os classificadores de forma e os classificadores de consistência. Também identificamos dois classificadores adicionais e contribuímos com o aprofundamento da descrição da semântica dos classificadores identificados por Mori $(2005,2007,2012)$.

CLASSIFICADORES DE FORMA: -tapa 'em forma de cacho, ramificado, volumoso'; -taku 'superfície plana', 'área', $-k a$ 'superfície larga', - $p i$ 'curvilinear/linear e saliente'; - $t \dot{t}$ 'semente, ou conteúdo de algo ou alguém'; -kana 'côncavo', -pana 'foliforme'. - pi 'formato grande e arredondado', -mepe 'amontoado saliente', -pe 'pastosa, cremosa, macia'.

CLASSIFICADORES DE CONSISTÊNCIA: -ja 'líquido', -pe 'pastosa, cremosa, macia'.

Esses morfemas têm como escopo o nome, razão pela qual são descritos como classificadores, seguindo Grinevald (2002) e Grinevald e Seifart (2004).

Exemplificando os classificadores do Mehináku: 
-tapa 'em forma de cacho, ramificado, volumoso'
1) ãhã-tapá
ser.genérico-Cl.cacho
'algo/pessoa volumosa'
6) maiki-tsapa milho- cl.cacho 'pirão de milho'
2) ata-tapá
pau-Cl.cacho
'raiz de árvore'
7) kahirí-tsapa embrulho-CL.cacho 'embrulho'
3) banana-tapá banana-CL.cacho 'cacho de banana'
8) nu-kati-tapa
1-perna-CL.cacho 'minha batata da perna'
4) juku-tapa urucum-CL.cacho
'cacho de fruto de urucum'
9) kujũ-tapa-j tipo .de.cesto-CL.cacho-abs 'saco escrotal'
5) halu-tapa fechado-CL.cacho 'beiju assado só de um lado'
10) nu-kana-tapa 1- côncavo-CL.cacho 'meu pulso'

O alomorfe -tsapa ocorre quando o tema precedente termina pela vogal $i$ :
11) kati-tsapa perna-CL.cacho
'batata da perna'
12) kahirí-tsapa embrulho-CL.cacho 'embrulho'

\section{-kana 'côncavo'}

13) ãhã-kaná algo- cl.côncavo 'coisa côncava'

14) ata-kana árvore-CL.côncavo 'canoa de tábua'

15) umaki-tfana trabalho-CL.côncavo 'equipamento de trabalho'

16) ata-pana-kana árvore-folha-cL.côncavo 'caixa de papelão'
17) amüna-kana frio-CL.côncavo 'roupa de frio'

18) uitfati-kana tipo de taquarinha-CL.côncavo 'cesto de uitfatü (tipo de taquarinha)'

19) tipihi-kana buraco-CL.côncavo 'buraco' 
-tit 'semente, ou 'conteúdo de algo ou alguém'

O morfema classificador $-t i$ tem três alomorfes fonologicamente condicionados: $-t i$, $-t s i$ e $t$ -

$\mathrm{O}$ alomorfe -tsi ocorre seguindo tema terminado pela vogal $i$ :

20) maiki-tsi

milho-CL.semente

'grão de milho'
22) ulei-tsi

plantação.de.mandioca-CL.semente 'mandioca'

21) i-wĩ-tsi

3-fígado-CL.semente

'fígado dele'

O alomorfe $t$ - ocorre precedendo o sufixo absolutivo -í, sendo resultado da queda da vogal $i$ precedendo o $i$ acentuado do referido sufixo.

$-\mathrm{ti}+$-í -> -ti

23) ka-t-í

perna-CL.semente-ABS

'perna'

28) tulũ-t-í

orelha-CL.semente-ABS

'brinco'

24) kana-t-1́

côncavo-CL.semente-ABS

'boca'

29) jana-t-í

pintura-CL.semente-ABS

'instrumento de pintar'

25) jesee-t-í

nádega-CL.semente-ABS

'nádega'

30) panaka-t-í

plantação-CL.semente-ABS

'muda'

26) kujũ-t-í

cesto-CL.semente-ABS

'testículo'

31) kanatapa-t-í

pulso-CL.semente-ABS

'pulseira'

27) hĩ-t-í

mama-CL.semente-ABS

'mamilo'

32) kalu-t-í

lágrima-CL.semente-ABS

'lágrima'

O alomorfe -ti ocorre nos demais ambientes:

33) mepe-ti

peixinho-CL.semente

'peixinho' 
34) piza-tí

pulga-CL.semente

'pulga'

35) nu-piza-ti-z̨a

1-pulga-CL.sementeMDP 'minha pulga'

36) taw-ti

ferro-cl-semente

'ferro'

37) sapi-ti

espinho.de.folha.de.buriti-CL.

semente

'espinho de folha de buriti'

38) n-ana-tí-za

1-pilão.de.pilão-CL.semente-mrn 'minha mão de pilão'

39) ata-ti

pau-CL.semente

'pauzinho'

40) wa-ti

coco.de.tucum-CL.semente

'coco de tucum'

41) nu-hese-ti-z̨a

1-amendoim-CL.semente-MDP

'meu amendoim'

42) kunu-ti

porta-CL.semente

'fechadura'

43) nu-kunu-ti-z̧a

1-porta-CL.semente- MDP 'minha fechadura'

44) julaka-ka-ti moqueado-perna-CL.semente 'jirau de moquear peixe'
45) julu-ti

torrado-CL.-semente

'milho torrado'

46) jue-ti castanha.de.pequi-CL.semente

'castanha de pequi'

47) melele-ti

tipo.de.coceira -CL.semente

'coceira'

48) nu-melele-ti

1- tipo.de.coceira.alérgica-CL.

semente

'minha coceira'

49) kupa-ti

peixe-CL.semente

'peixe'

50a) hayãka-tị

amassado-CL.semente 'cego'

50b) epu-ti

mutuca-CL.semente

'mutuca'

51) nu-kana-ti

1-côncavo-CL.semente

'minha boca'

52) nu-nihi-ti

1-carne-CL.semente

'minha carne'

53) nu-hi-tì

1-mama-CL.semente

'meu mamilo' 
-pi 'curvilíneo/linear e saliente'

54) nu-tua-pi-ra

1-esteira-CL.CURV- MDP .LINEAR-MDP

'minha esteira'

60) mami-pi

espião-CL.CURV.LINEAR

'espião'

55) kuja-pi

fio-CL.CURV. LINEAR-MDP

'barbante/linha Cléa'

61) wati-pi

tuCUM-CL.CURV.LINEAR

'colar de tucum'

56) nu-kuja-pi-ra

1-fio-CL.CURV .LINEAR-MDP

'meu barbante/ linha clea'

62) walu-pi

caramujo-CL.CURV.linear

'colar placa de caramujo'

57) taw-pi

ferro-CL.CURV. LINEAR

'cabo de ferro'

63) n-wãlũ-pĩ

1-caramujo-CL.CURV.LINEAR

'meu colar placa de caramujo'

58) nu-taw-pi-ra

1-ferro-CL.curv.LINEAR-MDP

64) wajala-pí

veia-CL.CURV.LINEAR-abs

'meu cabo de ferro comprido'

'veia'

59) ezuhi-niã-pi

anzol-fio.nom-CL.CURV.linear

'linha de pesca'

- $p \dot{t}$ 'formato grande e arredondado'

65) ãhã-pi

algo-CL.GRANDE/ARRED.

'algo grande'

67) aw-pi

eXC-CL.GRANDE/ARRED.

'panela grande n. 60'

66) kamalu-pi

argila-CL.GRANDE/ARRED.

'panela grande de barro'

68) ala-pi

aguapé-CL.GRANDE/ARRED.

'aguapé'

$-k a$ 'algo largo e plano'

69) ãhã-ká

algo-CL.LARGO

'coisa larga plana, achatada'

73) alata-ka

alumíno-CL.LARGO

'telha de alumínio' 
70) tana-ka-j

asa-CL.LARGO-ABS

'costas'

71) ata-ka

árvore-CL.largo

'tábua'

72) nu-tana-ka

1-asa-CL.largo

'minhas costas'
74) pawa-ka-j

rosto-CL.GRANDE/ARRED.-ABS

'rosto'

75) nu-pawa-ka

1-rosto-CL.largo

'meu rosto'

O alomorfe -t $\mathrm{fa}$ ocorre seguindo temas terminados por $i$.

76) ulei-tfa

mandiocal-CL.LARGO

'mandiocal'

77) enutfi-tya

trovão-CL.LARGO

'trovão'
78) mepehi-ta

cera.de.abelha-CL.LARGO

'cera de abelha'

-taku 'superfície plana', 'área'

79) enu-taku

em.cima-CL.SUP.PLANA

'céu'

80) ata-taku

pau-CL.SUP.PLANA

'floresta'

81) kehi-taku

terra-CL.SUP.PLANA

'chão'

82) weneku-taku

pátio.da.casa.dos.homens-CL.

SUP.PLANA

'pátio da casa dos homens'

83) hehé-teku

tacho-CL.SUP.PLANA

'superfície de tacho'
86) maiki-tsaku

milho-CL.SUP.PLANA

'milharal'

87) wati-taku

tucum-CL.cl. SUP. PLANA

'tucunzal'

88) alapi-taku

aguapé-CL.SUP. PLANA

'acampamento na lagoa de aguapé'

89) kitsapa-taku-j pé-CL.SUP.PLANA-ABS

'palma do pé'

90) nupana-taku-j

peito-CL. SUP.PLANA-ABS 'peito' 
84) epe-teku

capim-navalha-CL.SUP.PLANA

91) pawaka-taku-j

rosto-CL.SUP.PLANA-ABS 'rosto'

'capim-navalha'

85) mesa-taku

mesa-CL.SUP.PLANA

'superfície de mesa'

-mepe 'amontoado, saliente'

92) juhija-mepe-j

sombrancelha-CL.AMONTOADO-ABS

'sobrancelha'

94) nijẽ-mepe

fogo-CL.AMONTOADO

'cinza de meu fogo'

93) itsej-mepe

95) kusu-mepe-j

cinZa-CL.AMONTOADO

'um monte de cinza'

pubis-CL.AMONTOADO-ABS

'pubis'

\section{Consistência}

-ja 'líquido'.

96) aw-niza-já

exc-sangue-CL.LÍQUIDO

'que tem muito sangue'

97) kapizala-já

sopa.de.pimenta-CL.LíQUIDO

'sopa de pimenta'

105) kulata-já

quente-CL .LÍQUIDO

'água líquido quente'

106) katika-já

frio-CL. LÍQUIDO

'água gelada'

98) aw-putu-já

exc-coriza-CL. LíQUIDO

'coriza em excesso'

107) kaha-já

molhado-CL.LíQUIDO

'molhado'

99) kanawija-já

cana-CL LíQUIDO

'caldo de cana'

108) kati-pe-jé

caldo-CL.pastoso -CL. LíQUIDO

'caldo grosso'

100) awn-ihi-tya

exc-fezes-CL LíQUIDO

109) kehe-pe-jé

'que tem fezes em excesso'

espuma-CL.pastoso-CL.LíQUIDO

'líquido cheio de espuma' 
101) hĩjã-j-já

leite-abs-CL. LÍQUIDO

'leite'

102) aw-hapa-já

exc-barriga-CL. LíQUIDO

'barrigudo'

103) aruĩ-já

arroz-CL LÍQUIDO

'mingau de arroz'

104) uhu-já

batata-CL. LÍQUIDO

'mingau de batata'
110) nu -jana-la-ja

1-pintura-mrn-CL LíQUIDO

'minha tinta de pintar'

111) mukura-já

mandioca doce -CL.Ĺ́QuUDO

'caldo preparado de mandioca doce'

112) tipuka-ja

polvilho.diluído-CL.LíQUiDo

'mingau de polvilho diluído'

Os exemplos 108 e 109 mostram que um nome pode coocorrer com dois classificadores.

-pe 'pastosa, cremosa, macia'

113) pjulame-pe

trairão-CL.PASTOSO

'pirão de trairão'

114) pujte-pe

matrinxã-CL.PASTOSO

'pirão de matrinxã'

115) jajtsape-pe

tucunaré-CL.PASTOSO

'pirão de tucunaré'

116) walaku-pe

piau-CL.PASTOSO

'pirão de piau'

117) ihikume-pe

podre-CL.PASTOSO

'pirão de peixe podre'
121) tipe-pe

pedra -Cl.PASTOSO

'argila vermelha que serve de pintar'

122) n-ulu-le-pe

1-ralar-mrn-CL.PASTOSO

'minha massa de mandioca'

123) ule-pe

ular-CL.PASTOSO

'beiju'

124) n-aju-pe-le

1-algodão-CLPASTOSO -MRP

'meu algodão'

125) ije-pe

nuvem-CL.PASTOSO

'nuvem' 
118) hayãke-pe

amassado-CL.PASTOSO

'polvilho amassado para secagem'

126) malake-pe

raspado-CL.PASTOSO

'massa ralada'

119) jueti-pe

castanha.de.pequi-CL.PASTOSO

'pirão de castanha de pequi'

127) kehe-pé

espuma-CL.PASTOSO

'espumoso'

120) juluti-pe

torrado-CL.PASTOSO

128) aw-tiw-pé

'pirão com a mistura de milho

torrado socado'

exc-cabeça-CL.PASTOSO

'cabelo desajeitado ou enrolado'

$\mathrm{O}$ alomorfe -pje ocorre seguindo temas terminados por $i$ :

129) hali-pje

socado-CL.PASTOSO

'peixe socado'

130) n-akãj-pje-z̨e

1-pequi-CL.PASTOSO-MRP

'minha massa de pequi'
131) akãj-pje

pequi-CL.PASTOSO

'massa de pequi'

Parte dos classificadores tem origem claramente lexical, como são os casos de - $t \dot{\text { ‘ }}$ 'semente, grão', -ja 'líquido' e -taku 'superfície'. Os exemplos seguintes mostram as palavras fontes desses classificadores:

132) aju iti

algodão semente

'semente de algodão'

133) mimã itì

cabaça semente

'semente de cabaça'
134) melancia iti

melancia semente

'semente de melancia'

135) mamão iti

mamão semente

'semente de mamão'

Por outro lado, o classificador - $t i$ é uma forma reduzida da palavra para semente -iti que, enquanto lexema, pode ocorrer com a forma -iti ou -ti, e também -tsi, mas enquanto elemento classificador, ocorre sempre na forma $-t \dot{t}$ ou -tsi.

Observamos que o classificador -ja é originário do lexema -eja [eje] 'líquido', mas que, enquanto classificador, sua forma é -ja, o que nos permite considerar que essa forma é atualmente um morfema distinto da raiz -eja. 
ì-n-eja > in-eje /1-líquido/ 'líquido dele'

O exemplo que segue reforça mais ainda a natureza classificadora de -já, visto que se combina com a própria palavra para 'água':

136) uni-kulata-já

água-quente-CL.líquido

'água quente'
137) uni-katika-já água-fria-CL.líquido 'água fria/gelada'

Já o próximo exemplo evidencia a natureza derivacional dos classificadores:

138) itsei-ja

fogo-CL.líquido

'inflamáveis'
139) n-ahã-kana-paj

1-nome.gen-CL.côncavo-est

'eu estou corcunda'

Entretanto, não foi possível, até o presente, encontrar uma origem lexical para os classificadores -tapá, -pé e -pi.

\section{Os classificadores do Nahukwá-Kalapalo, do Suruí Paiter e do Mehinako e a tipologia linguística}

Grinevald e Seifart (2004:192) apresentam uma tipologia de sistemas de classificação nominal baseado em Grinevald (2000, 2002), que distingue três sistemas básicos: lexical (termos de classe e termos de medida), léxicogramatical (classificadores) e classes gramaticais (nominais/gênero), com sistemas intermediários, em termos de gramaticalização.

Os morfemas classificadores do Nahukwá-Kalapálo não se enquadram em nenhum dos tipos de classificadores descritos pelos autores supracitados. Embora sejam classificadores nominais, só classificam nomes possuídos, o que os distingue dos padrões conhecidos de classificação nominal. Há ainda um importante fato a ser observado, o de que os classificadores do Nahukwá-Kalapálo parecem únicos na família Karíb. Correspondem estruturalmente aos morfemas marcadores de posse das línguas dessa família (cf. Derbyshire, 1999:40-41), mas têm função classificadora. Já os nomes classificadores genéricos são comuns em línguas Karíb. Finalmente, a existência de classificadores de nomes em Nahukwá-Kalapálo o distingue da língua Kuikúro, na qual, os morfemas cognatos foram descritos como não possuindo semântica classificatória, mas marcando apenas classes morfológicas. ${ }^{9}$

\footnotetext{
9 "Para a flexão nominal, tradicionalmente chamada de sufixação de marcas de posse ou de relação ou dependência, temos um conjunto de formas alomórficas que competem para a inserção em um único morfema abstrato que carrega os traços de 'Posse' ou 'Relação' [REL]. Esses sufixos são distribuídos em Classes Flexionais Morfológicas” (Santos 2007:197).
} 
Quanto ao Suruí, temos um sistema de classificador com o menor número de distinções já reportado para as línguas nativas do Brasil e quiçá para as línguas da América do Sul. Embora nos limitemos a descrever o sistema de classificadores de uma única língua da família Mondé, o Suruí-Paitér, salientamos que encontramos fortes indicações de que um sistema análogo está ativo em Gavião, Zoró e Cinta-Larga e também na língua Tuparí, da família Tuparí.

Um fato a ser destacado é o de que o pertencimento de certos nomes a uma determinada classe pode não ser óbvio ou não ter sentido para um olhar externo, por exemplo, a palavra para avó em nahuká-Kalapálo ser marcada pelo classificador da classe dos capilares, peludos, que nascem como pelos.

Com este estudo chamamos a atenção para a necessidade de que as análises linguísticas considerem as fortes relações entre língua e cultura e o papel desta no delineamento da gramática de uma língua (cf. Everett 2012:196). Ressaltamos também a necessidade de que a tipologia dos sistemas de classificação nominal incorpore também os componentes semântico e pragmático, permitindo que se investigue como características semânticas, ou semântico-pragmáticas e gramaticais dos sistemas de classificação nominal interagem ou mapeiam um ao outro ${ }^{10}$.

\section{Referências}

Cabral, Ana Suelly A. C.; Suruí, Uraan; Tupari, Raul; Isidoro, Edineia. Classificadores em línguas Tupí de Rondônia, em uma perspectiva diacrônica (Em preparação)

Corbera Mori, Ángel H. 2005 "A posse nominal em línguas Arawak do sul e Arawak central: uma abordagem descritiva”. Estudos Linguisticos 43(1), pp. 236-268.

. 2007. Aspectos da estrutura nominal em Mehináku (Arawák). Estudos Lingüísticos (São Paulo), v. 1, pp. 249-257.

2012. Waurá e Mehináku: um breve estudo comparativo. Estudos Linguísticos (São Paulo. 1978), v. 41, pp. 196-205.

Crofts, M. 1973. Gramática Mundurukú. Brasília: SIL.

. 1985. Aspectos da Língua Mundurukú. Brasília: SIL.

Derbyshire, Desmond. 1999. Carib. In Dixon, R. M. W. and Aikhenwald, A. (eds.), The Amazonian Languages, pp. 23-64. Cambridge: Cambridge University Press.

Everett, D. 2012. Language: the cultural tool. New York: Pantheon Books.

Franchetto, B. 1995. Processos fonológicos em kuikúro: uma visão auto-segmental.

\footnotetext{
${ }^{10}$ Agradecemos a três leitores anônimos pela leitura cuidadosa e pelas críticas e sugestões feitas a este trabalho.
} 
In Leo Wetzels (org.). Estudos fonológicos das línguas indígenas brasileiras, pp. 53-84. Rio de Janeiro: Editora UFRJ.

Gabas Jr., N. 1999. A Grammar of Karo (Tupi, Brazil), Ph.D. dissertation, University of California, UEA.

Gomes. D. M. 2006. Estudo Morfológico e sintático da Língua Mundurukú (Tupí). Tese de Doutorado, Universidade de Brasília.

Grinevald, Colette. 2000. A morphosyntactic typology of classifiers. In: Systems of Nominal Classsification, Ed. Gunter Senft, Cambridge: Cambridge University Press. pp. 93-113.

Grinevald, Colette. 2002. Making sense of nominal classification systems. Noun classifiers and grammaticalization variable. In: Ilse Wischer \& Gabriele Diewald (Eds.). New reflections on Grammaticalization. Series: Typological studies in language, Vol. 49. Amsterdam/Philadelphia, John Benjamins B. V., pp. 259275.

Grinevald, Colette; Frank Seifart. 2004. Noun Classes in African and Amazonian languages: towards a comparison. Linguistic typology $\mathrm{n}^{\circ} 8, \mathrm{n}^{\circ} 2$, pp. 243-285.

Santos, Gélsama Mara Ferreira dos. 2007. Morfologia Kuikuro: Gerando nome e verbos. Tese de Doutorado, Universidade Federal do Rio de Janeiro.

Data recebimento: 25/03/2014.

Data aceite: $18 / 04 / 2014$. 